\title{
MANTENIMIENTO DEL ORDEN PÚBLICO: teoría, práctica y educación del policiamiento de los campeonatos europeos de fútbol de 2000 y $2004^{1}$
}

\author{
Otto M.J. Adang*
}

\begin{abstract}
As forças policiais diferenciam-se nos métodos de intervenção que utilizam para manter a lei e a ordem em grandes eventos que põem em perigo a ordem pública, a exemplo das partidas de futebol de alto risco e de certas manifestações públicas. Tradicionalmente, a ênfase no treinamento e desempenho policiais se coloca nas táticas de controle antidistúrbios e no uso de armas não letais, incluindo desde o cassetete e os produtos químicos até os canhões de água e as balas de borracha. A maior parte dos estudos geralmente deixam de lado a perspectiva policial e ignoram o fato de que os acontecimentos da ordem pública são processos intergrupais e uma consequência de interações em desenvolvimento. Neste artigo, apresentam-se os resultados de algumas pesquisas empíricas sobre a atuação da polícia na manutenção da ordem pública, que colocam em questão as perspectivas tradicionais do comportamento das massas.

PALAVRAS-CHAVE: ordem pública, policiamento de futebol jogos, táticas antimotim, a dinâmica das multidões, controle de multidões.
\end{abstract}

\section{INTRODUCCIÓN: investigación sobre la actuación policial para el mantenimiento del orden público}

Diferentes cuerpos policiales difieren en la manera en que preservan el orden público y los métodos de intervención que utilizan: el enfoque es, a veces, más preventivo y proactivo, y, en otras ocasiones, más represivo y reactivo, una distinción que es frecuentemente presentada en términos de actuaciones "blandas” o “duras” (Della Porta; Reiter, 1998 dan una perspectiva general sobre la actuación policial en protestas). Estas diferencias en los estilos de intervención policial se basan en la experiencia práctica antes que en los resultados de investigaciones sistemáticas sobre la actuación policial en el mantenimiento del orden público y sus efectos. Incluso en aquellos casos en que los entrenamientos y tácticas policiales se basan en la

${ }^{1}$ Traducción de Cecilia L. Ales, revisada por el autor.

* Psicólogo. Catedrático sobre Manutenção da Ordem Pública na Academia de Polícia dos Países Baixos. Professor visitante da Faculdade de Psicologia da Universidade de Liverpool.

De Kleiberg 15, 7312 SN, Apeldoorn. Postbus 1201, 7301 BL, Apeldoorn. otto.adang@politieacademie.nl investigación, suelen tener como sustento a teorías desactualizadas y desacreditadas. Al mismo tiempo, muchos estudios sobre la actuación policial en el mantenimiento del orden público suelen tener una limitada relevancia práctica. Son altamente teóricos o metodológicamente limitados de muchas maneras. La mayor parte de los estudios sobre mantenimiento del orden público se realizan después de que los hechos han ocurrido y el trabajo de campo es muy infrecuente. Además, tal como Stott y Reicher (1998a, 1998b) lo indican, los estudios disponibles a menudo soslayan la perspectiva policial e ignoran el hecho de que los sucesos de orden público son procesos intergrupales y, como tales, la consecuencia de interacciones en desarrollo. Así, en la actualidad, existe un conocimiento sistemático acotado en relación con los efectos que tienen los diferentes estilos de actuación policial sobre la ocurrencia o escalada del desorden público.

A partir de observaciones etológicas sistemáticas de 225 situaciones de disturbio en los Países Bajos, Adang $(1990,1991)$ concluyó que, contrariamente a la creencia popular y las teorías 
tradicionales sobre el comportamiento de masas, las multitudes no forman una masa homogénea con individuos que se comportan más o menos del mismo modo (véase también McPhail, 1991). Tampoco hay evidencias de que la gente reunida en grupos tenga una probabilidad mayor de usar la violencia que en cualquier otra circunstancia cotidiana, o que las multitudes muestren una mayor tendencia a comportarse de una manera que podría denominarse "emocional" o "irracional”. Las teorías tradicionales sobre las masas explican el comportamiento de las personas enteramente en términos de procesos internos al propio grupo y asumen que la patología de unos pocos agitadores induce y agudiza la patología de otros miembros del grupo (Stott; Reicher, 1998a). Los resultados a los que llegó Adang (1990, 1991) señalan la importancia de los procesos intergrupales y sugieren que es posible, y de hecho conveniente, que la policía se aproxime a las multitudes (tanto hinchas de fútbol como otros tipos de multitudes) con una actitud amigable pero firme, basada en la comunicación y la negociación, por una parte, (en este sentido la actitud podría ser considerada "blanda") y, por otra parte, que realice intervenciones tempranas frente a las transgresiones (en este sentido la actitud podría considerarse 'dura').

\section{LAEUROCOPA 2000}

In junio de 2000, el Campeonato Europeo de Fútbol (Eurocopa 2000) se realizó en los Países Bajos y Bélgica, países vecinos con diferente tipo de organización policial y diferentes culturas policiales. Sobre la base de experiencias prácticas e investigaciones previas (Adang, 1998) se definió el "Perfil del actuación policial en la Eurocopa 2000" de modo tal de unificar el trato con los visitantes, otorgar transparencia y promover una imagen positiva de las autoridades y los servicios policiales. En el perfil de actuación, se enfatizó que el contacto con la gente debía ser principal- mente preventivo y proactivo. La idea central subyacente a este perfil era que una actitud amigable pero firme por parte de la policía sería la más adecuada. Amigable, en el sentido de que los hinchas debían ser tratados como invitados. Firme, en el sentido de que se iban a mantener claros límites de conducta. Esto implicaría el empleo de pequeñas unidades de monitoreo vestidas con el uniforme ordinario, las cuales serían fácilmente contactables y, a la vez, se esperaba que contactaran activamente a los hinchas. Unidades descentralizadas de intervención (con sus elementos específicos, tales como vehículos antidisturbios, perros, carros hidrantes) se mantenían fuera de visibilidad mientras fuera posible. De acuerdo con el perfil de actuación, el policía ideal de la Eurocopa 2000 respetaría una serie de criterios, tales como contribuir con sus acciones a un alegre desarrollo de los acontecimientos, reconociendo situaciones potencialmente violentas sin temor a intervenir severamente, aunque sí de manera justa y oportuna.

Durante la Eurocopa 2000 se recolectó información de diversos tipos sobre las interacciones entre la policía y el público (Adang; Cuvelier, 2001). Un equipo internacional de supervisión integrado por cuatro funcionarios policiales experimentados visitó las ocho ciudades sede del campeonato al menos una vez. También se recolectó información por medio de funcionarios policiales extranjeros (spotters) que participaban en las operaciones. En cada una de las ocho ciudades sede, un equipo de observación integrado por al menos cuatro observadores (tanto estudiantes como funcionarios policiales) realizó observaciones sistemáticas y estructuradas durante los días de partido, asistió a las reuniones preparatorias y realizó entrevistas ad hoc a funcionarios policiales, guardias de seguridad e hinchas. El foco de las observaciones fuera del estadio en las ciudades sede estaba puesto en las interacciones entre la policía y el público. Las observaciones eran registradas en el momento con un grabador de mano.

El análisis de la información indicó que 
los conceptos tácticos aplicados en la práctica no se adecuaron consistentemente al concepto básico en las ocho ciudades sede. Fue posible distinguir dos estilos diferentes de actuación policial, a los que denominaré: actuación policial de alto perfil con una presencia policial relativamente alta (tres ciudades), y actuación policial de bajo perfil con menor cantidad de policías visibles (las otras cinco ciudades). Las ciudades que adoptaron el enfoque de "bajo perfil" tenían, en promedio, 10 policías visibles por cada 100 hinchas en las calles (en lugares en los que los hinchas se concentraban en grandes números). Ese es un número considerable de policías incluso si se lo compara con las prácticas policiales cotidianas y la regla en relación con el número de guardias de seguridad dentro de un estadio (1 guardia de seguridad por cada 100 hinchas). Sin embargo, existía una clara diferencia entre los dos estilos de actuación policial. La diferencia fue clara tanto en las observaciones sistemáticas de los equipos de observación en cada ciudad sede, como en las impresiones cualitativas de los spotters extranjeros (cada uno de los cuales trabajaba en más de una ciudad) y el equipo internacional de observación (que visitó cada una de las ocho ciudades sede y estaba en la mejor posición para realizar comparaciones). La diferencia también fue notada por los hinchas (Long, 2000).

La actuación policial en ciudades en las que se aplicó el estilo de "bajo perfil" estuvo más acorde con el "perfil de actuación". Las diferencias más sustantivas pudieron apreciarse en las siguientes cuestiones: los funcionarios policiales en ciudades con "bajo perfil” eran más fácilmente contactados por los hinchas, contribuían más a un festivo curso de los acontecimientos, mostraban mayor respeto por diferentes culturas y nacionalidades, desempeñaban un rol más preventivo, trataban a los visitantes como si fueran invitados y cambiaban entre distintos enfoques de actuación de manera más flexible cuando era necesario. Tanto en ciudades en las que la actuación policial tuvo un perfil alto como en aquellas en las que tuvo perfil bajo, los funcionarios policiales actuaron imparcialmente y sin prejuicios y, lo que no deja de ser destacable, no hubo diferencias significativas en la rigurosidad con la cual los funcionarios actuaron en ciudades con alto y bajo perfil (indicando que la actuación con bajo perfil no es sinónimo de una actuación "blanda" o que entraña una tolerancia ilimitada).

Las características del enfoque de "bajo perfil" consistían en un sustantivo, pero limitado, número de policías vistiendo el uniforme ordinario de fajina, patrullando en parejas o pequeños grupos, interactuando con los hinchas. Aunque estas interacciones eran amigables, las transgresiones de los hinchas eran respondidas con rapidez. Un informe de la Asociación Inglesa de Simpatizantes del Fútbol (English Football Supporters Association) (Long, 2000) afirma lo siguiente en relación con el enfoque de "bajo perfil": "La actuación policial fue excelente, y los hinchas disfrutaron de una cálida bienvenida de parte de las autoridades. Los policías eran tranquilos, educados, eficientes y amigables. Había una importante presencia policial en cada ciudad, pero ello no era intimidante o amenazante en modo alguno. Los policías estaban en las calles en grandes cantidades, en uniforme normal y a pie. Hacían un esfuerzo por hablar con los hinchas, a menudo riéndose y haciendo bromas, y su presencia contribuyó a que los hinchas se sintieran cómodos".

En el enfoque de "bajo perfil", el despliegue policial parecía basarse más en la inteligencia y la información proporcionada por los equipos de spotters. En el enfoque de "alto perfil" se podía ver a funcionarios policiales en las calles en un número superior al triple de aquellos visibles en las ciudades con "bajo perfil". Estos funcionarios estaban vestidos más a menudo con el equipo antidisturbios y acompañados por sus vehículos antidisturbios; formaban grupos más grandes, lo que hacía más difícil acercarse a ellos. Es importante destacar que la diferenciación hecha aquí es más sutil que la distinción entre 'tolerante' y 'represivo' (Della Porta; Reiter, 1998) o la de estilos 
militarizados versus estilos no militarizados de mantenimiento del orden público (Jefferson, 1994). Tanto en el enfoque de "alto perfil" como en el de "bajo perfil”, existía una clara estructura de comando y control y era posible recurrir a unidades de intervención con equipamiento antidisturbios.

La distinción entre la actuación policial de alto y bajo perfil era clara, pero no absoluta. Tanto en las ciudades con un enfoque de alto perfil como en aquellas con un enfoque de bajo perfil, con regularidad se podían distinguir elementos característicos del otro enfoque. En todas las ciudades sede, los funcionarios policiales actuaron imparcialmente y sin prejuicios. Tanto en ciudades con enfoques de alto como de bajo perfil, se llevaron adelante muchas iniciativas para recibir a los hinchas, informarlos y guiarlos hacia el centro de las ciudades y a los estadios. Coordinadores de hinchas, embajadas de hinchas, guardias de seguridad de la ciudad, entretenimientos, todo contribuyó a crear un clima hospitalario que fue complementado por el comportamiento policial. No hubo diferencias en la "rigurosidad" con la cual los funcionarios actuaron en ciudades con alto y bajo perfil. Sin embargo, en el enfoque de "bajo perfil”, los funcionarios policiales tendían a definir sus acciones más individualmente que en las ciudades con "alto perfil", lo cual podía ser desventajoso. El equipo internacional de observación también formuló una advertencia: pese a que el enfoque moderado, comunicativo, orientado al servicio funciona muy bien, podría ser difícil cambiarlo si la situación así lo requiere.

Contrariamente a lo que muchos esperaban, hubo sólo un número limitado de incidentes en las ciudades sede durante la Eurocopa 2000, la mayor parte de ellos breves y de una importancia menor. En vistas de ello y de la naturaleza mayormente festiva y segura del evento, puede afirmarse que el despliegue y el comportamiento policiales tuvieron el efecto esperado. Durante las observaciones sistemáticas, se registraba con intervalos de 15 minutos si había o no ocurrido un incidente durante los 15 minutos anteriores.
Este resultó ser el caso en el 10\% de las muestras $(\mathrm{N}=658)$, con alrededor de un tercio de incidentes pequeños, un tercio de incidentes de mediana intensidad y un tercio involucrando al menos a 10 personas. Llamativamente, el gran número de policías presentes en las ciudades con "alto perfil" no estuvo correlacionado con un número menor de incidentes. Podrían esperarse más incidentes en ocasión de llevarse a cabo partidos “de riesgo". Sin embargo, no fue tan simple como eso: en términos relativos, los incidentes fueron más frecuentes en las ciudades con "perfil alto" en los días en los que el riesgo no se vio incrementado! (Test de Chi-cuadrado, $\mathrm{P}<<.001$ ). Un examen más riguroso de los datos reveló que esta cifra podría atribuirse a los incidentes en sólo una de las ciudades con "perfil alto". Las observaciones cualitativas sugieren que, en esa ciudad (en oposición a otras), el accionar policial colectivo, materializado en el impedimento de paso o dispersión de hinchas, tendió a concretarse de manera previa a desatarse los incidentes, en lugar de ocurrir en respuesta a ellos.

Así, no hubo una correlación simple entre el enfoque o perfil adoptado y el número de incidentes. Sin embargo, los datos indicaron claramente que un mayor número de policías no necesariamente deriva en la ocurrencia de un menor número de incidentes. Si no responden a las transgresiones o no están presentes en lugares riesgosos, la influencia de un gran número de policías puede ser limitada. En vistas del número de funcionarios policiales desplegados (especialmente en las ciudades de "perfil alto"), resultó sorprendente el hecho de que, en numerosas ocasiones, ningún policía se encontrara presente en ciertos lugares pese a la presencia en el área de potenciales "causa-problemas". Por otra parte, un enfoque de "perfil alto" por sí mismo no causa o provoca incidentes. Sólo podría incrementarse la posibilidad de ocurrencia de incidentes violentos si un enfoque de "perfil alto" es acompañado por métodos de actuación policial más agresivos. Éste pareció ser el caso en una de las ciudades sede. En esta misma 
ciudad, la estructura de comando y control aplicada difería de aquellas utilizadas en las restantes ciudades y los análisis binacionales de riesgos no fueron puestos en práctica.

Antes de la Eurocopa 2000, el perfil de actuación policial recibió críticas por parte de algunos funcionarios policiales. En su opinión, el perfil de actuación establecía lo obvio y simplemente describía la conducta policial cotidiana. Para ellos, el énfasis en el perfil de actuación policial era un insulto: implicaba que se les debía decir cómo hacer su trabajo correctamente, porque el modo en que solían hacerlo aparentemente estaba mal. El hecho de que, en términos generales, el comportamiento policial soliera estar acorde con el perfil de actuación indica que, para muchos policías, este perfil probablemente no se desviaba dramáticamente de lo que hacían diariamente. Sin embargo, los resultados de este estudio indican que el comportamiento policial no se correspondió exactamente con el perfil de actuación, ni en las ciudades con "bajo perfil" ni en aquellas con "alto perfil”. No puede sorprendernos lo que ello indica: para que ocurra un cambio de actitud, se requiere más de un día de entrenamiento. Aplicar el perfil de actuación policial implica más que hacer que cada funcionario policial utilice correctamente el mismo uniforme. Además, definir un perfil de actuación puede ser visto como un ejemplo de liderazgo claro: al adoptar y promover un perfil de actuación los oficiales a cargo dan una clara indicación de cómo quieren ellos que sus policías se comporten.

Por supuesto, la actuación policial es sólo uno de los muchos factores relevantes que influencian el desarrollo y escalada de incidentes de orden público. Otro importante factor que influencia el orden público y la seguridad de los partidos de fútbol es la motivación de los individuos que están involucrados (Adang, 1998). En relación con lo anterior, en la mayor parte de los países, los hinchas que siguen a la selección nacional usualmente suponen una amenaza menor al orden público que aquellos que siguen a los equipos locales. Ello en sí mismo limita el número de provocadores potenciales que asisten a eventos tales como la Eurocopa. 2000. Una ayuda adicional provino del hecho de que los seleccionados nacionales, cuyos seguidores son más violentos, quedaron fuera de la competencia después de la primera ronda. Adicionalmente, a través del esfuerzo de cooperación policial internacional, muchos potenciales provocadores ni siquiera llegaron a los Países Bajos o a Bélgica, porque fueron persuadidos de quedarse en casa o bien detenidos en la frontera. Estas circunstancias ampliaron las posibilidades de implementar un enfoque orientado a la hospitalidad, amigable pero firme.

En términos generales, podría concluirse que el despliegue policial contribuyó a que el evento transcurriera en un clima festivo y seguro. La evaluación oficial encargada por el gobierno holandés llegó a la misma conclusión (COT, 2000). Hubo sólo un número limitado de incidentes en las ciudades sede, la mayor parte de ellos breves y de menor importancia. Hubo una clara distinción entre dos tipos de despliegue policial: "actuación de alto perfil”, con gran número de policías, parte de ellos con equipamiento antidisturbios, y una "actuación de bajo perfil", en términos relativos, más acorde con el "perfil de actuación" definido antes del comienzo del campeonato. Sobre la base de los datos recolectados, podrían identificarse varios elementos de "buena práctica". Entre ellos se incluyen los siguientes:

- Definición de un perfil de actuación policial claro.

- Comunicación y entrenamiento para implementar el perfil de actuación policial.

- Atención recurrente al perfil de actuación durante las reuniones preparatorias.

- Contacto activo con los hinchas (obtención de información, construcción de una relación).

- Presencia policial fuertemente dependiente del relevamiento de información y el análisis de riesgos.

- Patrullaje policial en pareja o en pequeños grupos. - Presencia policial con clara visibilidad, 
destacándose entre la multitud.

- Clara comunicación de los límites y actuación frente a su incumplimiento.

- Líneas de comando cortas y claras.

Un grupo de trabajo sobre desorden en el fútbol (2001) en el Reino Unido concluyó, en un informe remitido al Parlamento, que el tipo de actuación de "bajo perfil" proporciona un modelo cuya aplicación debería fomentarse a lo largo de Europa. El grupo de trabajo sostuvo que las tácticas policiales imaginativas y focalizadas empleadas han sido universalmente elogiadas, incluso por los propios hinchas. El grupo de trabajo del Reino Unido sobre desorden en el fútbol también recomendó que se profundice la investigación sobre el impacto de la policía y otras tácticas de seguridad pública sobre el comportamiento de los simpatizantes.

Es importante enfatizar que el estilo de actuación "amigable pero firme" no es sinónimo de 'dejar hacer': implica que los funcionarios policiales respondan activamente, en los momentos iniciales, a infracciones relativamente menores a los límites pre-fijados. Los resultados muestran que es posible mantener el orden público de manera efectiva (y eficiente, considerando el hecho de que se requieren menos policías) de este modo, sin crear un estado de sitio ni alterar las actividades de la gente involucrada (en este caso, los hinchas). Un elemento clave en el enfoque "amigable pero firme" es la presencia de funcionarios policiales en parejas o en pequeños grupos, que resulten accesibles para los hinchas e incluso contacten activamente a estos últimos. Es posible obtener información sobre las intenciones, la información puede ser intercambiada más fácilmente, el anonimato de los hinchas se reduce y se puede construir una relación temporaria. Al mismo tiempo, los límites son claramente comunicados y hechos respetar en etapas iniciales, reduciendo así la necesidad de recurrir a tácticas de confrontación colectiva.

De este modo, la policía está mejor preparada para discriminar entre la gente involucrada y se podría evitar la gestación de un antagonis- mo interno-externo al grupo entre gente que se reúne (en este caso turistas e hinchas) y la policía. Desde una perspectiva psicológica, Stott y Reicher (por ej. 1998a) indican que, cuando una multitud inicialmente heterogénea es tratada por la policía como un todo homogéneo, ello ha llevado a los integrantes del grupo a reconceptualizarse como miembros de una categoría común, iniciando así un ciclo de tensión y agravando el conflicto. Es este bien conocido mecanismo de dentro-fuera del grupo el que juega un rol tan importante en la ocurrencia y escalada de incidentes de violencia colectiva. (Adang, 1999).

\section{PRINCIPIOS PARA EL MANTENIMIENTO DEL ORDEN EN GRANDES MANIFESTACIONES}

El perfil de actuación definido para la Eurocopa 2000 se ajusta muy bien a cuatro principios para el mantenimiento del orden en grandes manifestaciones según fueran formulados por Reicher y otros (2004). De hecho, este documento fue escrito sobre la base del estudio de la Eurocopa 2000 y la investigación científica de la dinámica de grupos (por ej. Stott; Reicher, 1998a, 1998b). El punto de partida teórico para estos principios es el modelo elaborado de identidad social (ESIM), que apoya la idea de que el 'desorden' colectivo es posible gracias a la prominencia psicológica compartida de una identidad social común entre los participantes del grupo (Reicher, 1984, 1987). Las dimensiones definitorias de esta identidad sirven para explicar los límites normativos de la acción colectiva (lo que la gente hace), y el grado de participación (quiénes participan y quiénes no) en un evento grupal. Este análisis de la 'identidad social' sostiene que la dinámica de la interacción entre grupos es un componente esencial de la psicología del ‘desorden’ generalizado. Para contribuir a prevenir el desorden generalizado, Reicher y otros (2004) identifican cuatro principios básicos para la actuación policial en grandes manifestaciones: 
educación, facilitamiento, comunicación, y diferenciación, términos que definen del siguiente modo.

Educación: Como los grupos actúan sobre la base de identidades sociales, y como las multitudes están comúnmente formadas por varios grupos, resulta de gran importancia práctica informarse y conocer las identidades sociales de los diversos grupos que integran la multitud: sus valores y estándares, sus fines y objetivos, su sentido de lo que es correcto y está bien, sus estereotipos y expectativas de otros grupos, su historia de interacción con estos grupos y cualquier otra cosa (fechas, lugares, objetos, formas de acción) que tenga particular importancia en términos simbólicos.

Las actividades de inteligencia no sólo deben tener por objetivo a los individuos violentos y sus planes, sino que también deben estar dirigidas a comprender las identidades sociales de los grupos que se reúnen, a conocer cuáles son sus objetivos, a decidir si y - en tal caso cómo apoyarlos, a conocer qué formas de actuación policial podrían antagonizarlos y generar mayor simpatía con los elementos violentos en la multitud. El mismo esfuerzo que se realiza para identificar a los individuos violentos debe realizarse para comprender la identidad del grupo. Asimismo, debe otorgarse similar relevancia a ambos factores en las reuniones preparatorias de inteligencia.

Facilitar: En lugar de evitar que la gente haga lo que desearía y, por lo tanto, frustrarla, en todas las etapas de la intervención policial, debe ponerse el énfasis en el facilitamiento. Al planificar un evento, se deben identificar los fines legítimos de los miembros de la multitud, de modo de poder organizar la intervención policial de manera tal que permita alcanzar dichos fines. Si existe una razón por la cual ellos no pueden alcanzarse, no se trata simplemente de dar una respuesta negativa, sino de ser creativos $\mathrm{y}$ encontrar modos alternativos de alcanzar (y que se note que se alcanzan) dichos fines. Si el peligro de que exista violencia o la efectiva ocurrencia de hechos de violencia fuerza a la policía a imponer límites a la multitud, es fundamental dejar en claro por qué ha sido necesario imponer dichos límites y proporcionar medios alternativos a través de los cuales los fines puedan alcanzarse. Todo esto es lo que hace que la comunicación sea importante (ver más adelante).

La tentación de controlar y ponerse duros puede ser mayor en particular cuando la violencia irrumpe. Es en este punto en que una clara indicación de que la policía está apoyando los fines colectivos - y que ellos están siendo puestos en riesgo por la violencia - puede hacer la diferencia entre la escalada y la desescalada. Por supuesto, para que ello ocurra, no sólo es necesario que la policía esté tratando de facilitar la realización de los fines de la multitud, sino también que la multitud lo note. De nuevo, esto señala la importancia de la comunicación.

Comunicación: Una de las paradojas de la intervención policial en grandes concentraciones de gente es que la importancia de la comunicación con la multitud es directamente proporcional a la necesidad de evitar relaciones potencialmente conflictivas. Sin embargo, en situaciones en las que las relaciones son potencialmente conflictivas, la multitud es menos propensa a confiar en lo que la policía tiene para decir. El mero intento de informar a la multitud, cómo se ha diseñado la intervención policial para facilitar la manifestación, puede ser visto como algo deshonesto e incrementar la hostilidad, especialmente cuando hay una larga historia de conflicto. Por ello también es importante cómo se lleva a cabo la comunicación. Es mejor recurrir a gente respetada por grupos que integran la multitud y en la que confían, preferentemente 'uno de los suyos'. Por supuesto, podría ser imposible encontrar a figuras que sean aceptadas por todos los grupos que componen la multitud. Sin embargo, resulta crítico que ellas no se hayan 'autoconvocado' o se las haya elegido por sus vínculos con las autoridades, sino que sean consideradas representativas por sectores significativos de la multitud (una vez más, el conocimiento 
cultural de los grupos es crítico en este sentido). Para ser realmente efectivos, estos comunicadores o mediadores deben estar disponibles y activos en momentos de violencia incipiente, bien desde el comienzo (o incluso antes de su ocurrencia). Es una observación reveladora del equilibrio entre las estrategias represivas y facilitadoras de la intervención policial en grandes concentraciones el hecho de que existen grandes reservas de vehículos blindados, filas de policías con cachiporras y demás elementos que pocas veces son utilizados, mientras que se presta mucha menos atención a las tecnologías comunicativas. Grandes pantallas y sistemas de comunicaciones móviles podrían ser más útiles en grandes concentraciones que los camiones hidrantes.

Diferenciación: Es importante ser concientes de las diferentes identidades de los grupos que se reúnen y de sus diferentes maneras de actuar y reaccionar. Resulta aún más importante actuar sobre esa base y no tratar a todos los miembros de la multitud como si fueran iguales. Una distinción básica que debe hacerse es la diferenciación entre la gente en virtud de lo que realmente está haciendo, antes que en base a las categorías a las cuales (supuestamente o no) pertenece. Es precisamente cuando algunos miembros de la multitud comienzan a ponerse hostiles que se torna importante tratarlos de manera amigable. Es precisamente para frenar la violencia de unos pocos que se debe ser permisivo con la mayoría. Es difícil, porque la gente tiende a tratar a otros grupos como homogéneos, siendo que, si algunos entre ellos actúan negativamente, todo el mundo es visto negativamente (por ej. Judd; Park, 1988). También es difícil porque los costos que implica para el policía (que está en la primera fila) tratar a alguien con confianza cuando es realmente hostil son más grandes que los costos de tratarlos con hostilidad cuando realmente son confiables. Para estar en condiciones de discriminar, se requiere desarrollar tácticas más diferenciadas que las que usualmente se emplean, y la diferenciación es una consideración que debe tenerse en cuenta en toda decisión táctica o estratégica, en el entrenamiento, la planificación, el equipamiento y la operación en grandes concentraciones de gente.

\section{EUROCOPA 2004}

Estos principios fueron subsecuentemente utilizados por la policía nacional portuguesa (PSP) en su preparación para la edición 2004 del Campeonato Europeo de Fútbol que se llevó a cabo en Portugal (Eurocopa, 2004). Se adoptó un enfoque estratégico que estuvo en línea con el enfoque de "bajo perfil". A pedido de la PSP y con el financiamiento del Consejo de Investigaciones Económicas y Sociales (ESRC), la Universidad de Liverpool y la Academia de Policía de los Países Bajos realizaron conjuntamente un estudio independiente sobre el comportamiento de los hinchas y el mantenimiento del orden público por parte de la policía durante la Eurocopa 2004 (Adang; Stott, 2004; Stott; Adang, 2005). La investigación se abocó al estudio de tres cuestiones específicas. En primer lugar, comprender los procesos psicológicos y la dinámica entre grupos subyacentes tanto a la presencia como a la ausencia de 'desorden' en el contexto del fútbol internacional. En segundo lugar, evaluar la efectividad de las estrategias policiales utilizadas para prevenir el desorden de las multitudes. En tercer lugar, desarrollar la relación entre la ciencia y la práctica en el campo de la intervención policial para el mantenimiento del orden público, proporcionando un enfoque sustentado en la observación empírica para la planificación de la seguridad en futuros campeonatos internacionales de fútbol.

El proyecto combinó dos enfoques metodológicos de observación estructurada y etnografía para recolectar datos sobre los policías y los hinchas durante el evento. La observación estructurada empleada fue similar a la utilizada en el estudio de la Eurocopa 2000, permitiendo hacer comparaciones directas. (Abrir nota de pé 
de página e colocar estas informações sobre a pesquisa na mesma: Entre el 12 de junio y el 4 de julio, se reunió una cantidad de información (además de documentación por escrito): entrevistas a funcionarios policiales portugueses $\mathrm{y}$ extranjeros;entrevistas a hinchas, especialmente de Alemania, Inglaterra y los Países Bajos; respuestas de hinchas ingleses, alemanes y holandeses a un cuestionario colgado en la web; entrevistas a miembros de proyectos de hinchas o embajadas de hinchas; observaciones estructuradas en ciudades sede, en las tardes previas a los partidos y los días de partido, por parte de equipos de observación entrenados (cuatro equipos con cuatro observadores cada uno);observaciones semi-estructuradas por parte de un equipo internacional de supervisión y los líderes del proyecto.)

Se seleccionaron catorce partidos para realizar las observaciones, a saber: todos los partidos de las selecciones alemana e inglesa (considerados partidos de riesgo por las autoridades portuguesas) y partidos de las selecciones de los Países Bajos y Portugal (considerados partidos sin riesgo elevado por las autoridades portuguesas). Los resultados indican que:

1. Cuando la policía tenía una presencia visible, la proporción de policías visibles era en promedio de 4 funcionarios por cada 100 hinchas, tanto en situaciones normales como de riesgo. Esta cifra es un poco menor, pero, sin embargo, comparable a la de la presencia visible en circunstancias de bajo perfil y riesgo normal en la Eurocopa 2000 (en promedio 6 policías por cada 100 hinchas). No hay diferencias entre la actuación policial de alto y bajo perfil en la Eurocopa 2004, mientras que sí había una clara diferencia en este sentido durante la Eurocopa 2000. Se debe tomar nota del hecho de que, en Portugal, se hizo un uso extensivo de policías vestidos de civil, quienes estaban desplegados dondequiera que los hinchas se reunieran en grandes números.

2. Si la policía estaba presente, ello no era en la forma de policía antidisturbios completamente equipada. Durante la Eurocopa 2000, la policía antidisturbios, con todo su equipamiento, estaba presente en aproximadamente $15 \%$ de las muestras, con una presencia significativamente mayor en situaciones de perfil alto y cuando los análisis de riesgo indicaban un riego incrementado. Durante la Eurocopa 2004, no hubo una diferencia significativa entre situaciones de alto y bajo riesgo en relación con la visibilidad de la policía antidisturbios. Se debe tomar nota de que los observadores indicaron que las unidades de policía antidisturbios estaban de hecho presentes en los alrededores de los lugares en los que los hinchas se reunían: sin embargo, estaban ubicadas de tal manera que no eran directa o fácilmente visibles para los hinchas.

3. Durante la Eurocopa 2004, casi no se registraron incidentes durante la observación estructurada (sólo en 0,4\% de todas las muestras). Durante la Eurocopa 2000, 10\% de las muestras tenían incidentes, ocurriendo la mayor parte de ellos en situaciones de riesgo normal y alto perfil (indicando el efecto contraintuitivo que puede tener la actuación policial de alto perfil: en lugar de disuadir la ocurrencia de incidentes, puede incluso provocarlos).

Mientras que la Eurocopa 2000 fue considerada un éxito por los países organizadores, debido a la baja frecuencia de incidentes y porque la muy anticipada 'guerra de hinchadas' no se concretó, la Eurocopa 2004 se caracterizó por una casi total ausencia de incidentes. Tal como también fue el caso durante la Eurocopa 2000, más allá de unos pocos intentos individuales de invadir el campo de juego, no ocurrieron incidentes dentro de los estadios. El análisis de los datos obtenidos en esta evaluación independiente permitió concentrarse en la causa de la baja frecuencia de incidentes ocurridos en Portugal. El hecho de que en particular Alemania y el Reino Unido hayan impedido que conocidos barras bravas viajaran a Portugal seguramente fue un factor. Sin embargo, tanto las observaciones como 
las entrevistas con equipos de policías extranjeros indicaron que los individuos conocidos como 'barrabravas' o autodenominados tales estaban de hecho presentes en Portugal. Observaciones de los escasos y acotados incidentes que sí ocurrieron, así como también de incidentes potenciales que tenían todos los ingredientes para escalar pero que de hecho no lo hicieron, permiten afirmar que, pese a la baja visibilidad de la presencia policial, la mayor parte de estos incidentes fueron respondidos rápidamente. Así, la ausencia de incidentes mayores no fue sólo una cuestión de suerte. Las rápidas y focalizadas intervenciones policiales de bajo perfil impidieron la escalada. De este modo, se fijaron claros límites de conducta. Las estrategias y tácticas policiales contribuyeron a una atmósfera en la cual los fanáticos se identificaban primero como hinchas del fútbol o de la Eurocopa 2004 (antes que sólo como hinchas de sus respectivas selecciones nacionales), el comportamiento no violento fue la norma y los hinchas opuestos a la violencia resultaron fortalecidos. Como consecuencia, se pudieron observar numerosos ejemplos de autocontrol entre los propios hinchas.

Este argumento es fortalecido por los incidentes que sí ocurrieron en el Algarbe (en Albufeira), en donde las tácticas policiales fueron diferentes. Inicialmente, no se fijaron límites de conducta y la respuesta policial al comenzar los incidentes no diferenció entre quienes causaban problemas y terceras personas. Aquí, fueron los que querían usar la violencia quienes resultaron fortalecidos. La conclusión de la investigación (Adang; Stott, 2004; Stott; Adang, 2005) fue que la estrategia y las tácticas policiales (el enfoque de bajo perfil, en el que los límites de conducta son fijados de manera firme y amigable) fueron exitosas y contribuyeron a crear una identidad pro fútbol/Eurocopa 2004 común entre los hinchas y el fortalecimiento de la no violencia y el autocontrol por parte de ellos. Por supuesto, otros factores tales como la hospitalidad y el comportamiento de los hinchas también contribuyeron a esto.

\section{LA RELACIÓN ENTRE TEORÍA, PRÁCTICA Y EDUCACIÓN}

Las experiencias de la Eurocopa 2000 y la Eurocopa 2004 ilustran cómo la teoría, la práctica y la educación pueden beneficiarse mutuamente una de la otra. La estrategia y las tácticas se basaron en el conocimiento disponible y este conocimiento fue también empleado para preparar a los policías para el campeonato. Las experiencias relatadas en este documento también señalan el hecho de que adherir a una clara distinción entre la investigación dura y la investigación aplicada es fútil: la relación entre la investigación y la práctica permitió que se llevaran a cabo estudios innovadores con valor tanto teórico como práctico. Para el futuro de la educación policial, será cada vez más importante incorporar el conocimiento disponible e involucrar a investigadores sobre temas policiales en el desarrollo de la currícula. Al mismo tiempo, se necesita más trabajo de campo comparativo sobre buenas prácticas y se deben realizar mayores intentos por vincular a la investigación sobre cuestiones prácticas del trabajo policial con conceptos teóricos. Siempre que ello sea posible, la investigación debe hacerse en el campo (y los investigadores sobre temas policiales deberían familiarizarse con el trabajo policial). Debe reconocerse que los investigadores mantienen su independencia y los funcionarios policiales no son las personas adecuadas para formularse las preguntas de investigación.

(Recebido para publicação em julho de 2010) (Aceito em setembro de 2010)

\section{REFERÊNCIAS}

ADANG, O.M.J. Geweld en politie-optreden in relsituaties. Utrecht: Universiteit Utrecht, 1990.

Gedrag van mensen tijdens rellen. In: DEFARES, P.B.; VAN DER PLOEG, J.D. (Ed.) Agressie. Van Gorcum: Assen, 1991.

Hooligans, autonomen, agenten: eweld en politieoptreden in relsituaties. Samsom, Geneva: Amsom, 1998. 
Systematic observations of violent interactions between football hooligans. In: THIENPONT, K.; CLIQUET, R. (Ed.) In-group/ out-group behaviour in modern societies: an evolutionary perspective . Vlaamse Gemeenschap: Brussel, 1999

CUVELIER, C. Policing Euro 2000. Apeldoorn: Dutch Police Academy, 2001.

;. STOTT C. Evaluation of public order policing during Euro 2004. Tijdschrift voor Veiligheid en Veiligheidszorg, n.4, p.46-47, 2004.

COT. Evaluatie EK 2000 - openbare orde en veiligheid. Samsom,Geneva: Alphen aan den Rijn, 2000.

DELLA PORTA, D.; REINER, H. The policing of protest in contemporary democracies: an introduction. In:

(Ed.) Policing protest. Minneapolis: University of Minnesota Press, 1998.

JEFFERSON, T. The case against paramilitary policing. [S.l.]: Open University Press; Milton Keynes, 1994.

JUDD, C.M.; PARK, B. Out-group homogeneity: judgments of variability at the individual and group levels. Journal of Personality and Social Psychology, v.54, jun., p.778-788, 1988.

LONG. EURO 2000. Policing, arrests and deportations A report by the Football Supporters' Association. Liverpool: Football Supporters Association, 2000.

MCPHAIL, C. The myth of the madding crowd. New York: Aldine de Gruyter, 1991.
REICHER, S.D. The St. Paul's riot: an explanation of the limits of crowd action in terms of a social identity model. European Journal of Social Psychology, v.14, p.1-21, 1984.

Crowd behaviour as social action. In: J.C. TURNER, M.A.; HOGG, P.J.; OAKES; REICHER, S.D.; WETHERELL, M.S. Rediscovering the social group: a selfcategorization theory. Oxford: Blackwell, 1987.

; STOTT, C.; CRONIN, P., y Adang, O an integrated approach to crowd psychology and public order policing Policing: an International Journal of Police Strategies and Management. v.27, p.558-572, 2004.

STOTT, C.J.; O.M.J. Adang. Crowd dynamics, policing and 'hooliganism' at 'Euro2004'. End of award report, ESRC, 2005. <http:/www.esrcsocietytoday.ac.uk/ESRCInfoCentre/ ViewOutputPage aspx?data=kWyXVzYZdoUz29Q2xb9GbrLk cCvvu88YjV3rVv9QxLd3B CrHP4aXD8auqhkRYxhBOBS NWWise2uGQOtn7jia\%2fsygQXNREIXhYUsW\%2bZUP pj zJxveaBft2hORVd5UEc4\%2fX3ab2js3Gkx8K9BU599yiQTe unIbVPJ\%2bLU33HAw2wjs6 BWpuQol5sEv1nMfU8CNu2 Rlnc\%2fBk0Yw0\%3d\&xu=\&isAwardHolder=\&isProfiled =\& Award HolderID $=\&$ Sector $=>$.

STOTT, C. J.; REICHER, S. Crowd action as intergroup process: Introducing the police perspective. European Journal of Social Psychology, New York, n.26, p.509-529, 1998a.

How conflict escalates: the inter-group dynamics of collective football crowd 'violence'. Sociology, [S.l.] v.32, p.353-377, 1998b.

WORKING GROUP ON FOOTBALL DISORDER. In: LORD BASSAM (Chaired) Report and recommendations. London: Home Office, 2001. 
MAINTENANCE OF PUBLIC ORDER: theory, practice and teaching of the police for the European Football Championship in 2000 and 2004

\section{Otto M.J. Adang}

Police forces differ in the methods of intervention used to maintain law and order in major events that endanger public order, like high risk soccer matches and certain public demonstrations. Traditionally, the emphasis on police performance and training is put on riot control tactics and use of nonlethal weapons, from batons and chemicals to water cannons and rubber bullets. Most studies generally fail to consider the perspective of the police and ignore the fact that the events of public order are intergroup processes and a consequence of interactions in development. In this paper, we present the results of some empirical research on the role of police in maintaining public order, which put into question the traditional perspectives of behavior of the masses.

KEYwORDs: public order, policing of football matches, riot control tactics, dynamics of crowds, crowd control .
MAINTIEN DE 'L'ORDRE PUBLIC: théorie, pratique et formation de la police pour le Championnat Européen de Football en 2000 et 2004

\section{Otto M.J. Adang}

Les forces de police s'exercent de manière différente en fonction des méthodes d'intervention utilisées pour maintenir l'ordre et faire respecter la loi dans les grandes manifestations qui mettent en danger l'ordre public, tels les matchs de football à haut risque et certaines manifestations publiques. Traditionnellement, l'accent est mis sur la formation et les performances de la police quant aux tactiques de contrôle antiémeutes et l'utilisation d'armes non létales, y compris les bâtons et les produits chimiques, les canons à eau et les balles en caoutchouc. La plupart des études ne tient généralement pas compte du point de vue de la police et ignore le fait que les événements dus à l'action de l'ordre public sont le résultat d'un processus intergroupes et la conséquence d'interactions en développement. Dans cet article, nous présentons les résultats d'une série de recherches empiriques sur le rôle de la police pour le maintien de l'ordre public, qui remet en question l'approche traditionnelle du comportement des masses.

Mots-CLÉS: ordre public, surveillance policière des matchs de football, tactiques antiémeutes, dynamique des foules, contrôle des foules.

Otto M.J. Adang - Psicólogo. Catedrático da disciplina Manutenção da Ordem Pública na Academia de Polícia dos Países Baixos. Professor visitante da Faculdade de Psicologia da Universidade de Liverpool. Responsável pelo projeto de pesquisa Manejo de Situaciones Peligrosas, cujo eixo são as interações entre a polícia e o público em situações potencialmente violentas. Desenvolve pesquisas em numerosos países europeus e foi consultor das polícias de Argentina, Bélgica, Países Baixos, Alemanha, Irlanda do Norte, Portugal e Sudáfrica. É autor de inúmeros artigos e livros sobre a temática, publicados na Europa. Publicou recentemente, em espanhol, o artigo "La mejor policía del mundo aprende a cualquier precio". Cuadernos de Seguridad, Buenos Aires n. 9, p. 47-62, 2009. 